Comparison of the imaging performances for recently developed monolithic scintillators: CRY018 and CRY019 for dual isotope gamma ray imaging applications

This content has been downloaded from IOPscience. Please scroll down to see the full text. 2017 JINST 12 C01055

(http://iopscience.iop.org/1748-0221/12/01/C01055)

View the table of contents for this issue, or go to the journal homepage for more

Download details:

IP Address: 151.100 .142 .85

This content was downloaded on 19/05/2017 at 11:15

Please note that terms and conditions apply.

You may also be interested in:

Investigation of radiation detection properties of CRY-018 and CRY-019 scintillators for medical imaging

R. Pani, M. Colarieti-Tosti, M.N. Cinti et al.

Study of the characteristics of a scintillation array and single pixels for nuclear medicine imaging applications

Zhu Jie, Ma Hong-Guang, Ma Wen-Yan et al.

Imaging characterization of a new gamma ray detector based on CRY019 scintillation crystal for PET and SPECT applications

C. Polito, R. Pani, C. Trigila et al.

Measuring the $14 \mathrm{C}$ content in liquid scintillators

T Enqvist, I R Barabanov, L B Bezrukov et al.

A newly developed wrapping method for scintillator detectors

L Stuhl, A Krasznahorkay, M Csatlós et al.

A crystal identification method for monolithic phoswich detectors based on scintillation light distribution

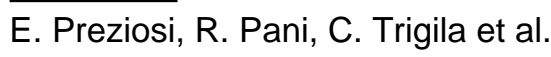

Performance study of a PET scanner based on monolithic scintillators for different Dol-dependent methods

E. Preziosi, S. Sánchez, A.J. González et al.

Timing scintillation detector with SiPM incorporated throughout a scintillator's body

E V Antamanova, P Zh Buzhan, V A Kaplin et al. 
$18^{\text {TH }}$ INTERNational Workshop on Radiation IMAging Detectors

3-7 JULY 2016,

Barcelona, Spain

\title{
Comparison of the imaging performances for recently developed monolithic scintillators: CRY018 and CRY019 for dual isotope gamma ray imaging applications
}

\author{
C. Polito, ${ }^{a}{ }^{1}$ R. Pani, ${ }^{b}$ C. Trigila, ${ }^{c}$ M.N. Cinti, ${ }^{d}$ A. Fabbri, ${ }^{d}$ V. Frantellizzi,${ }^{e}$ G. De Vincentis, ${ }^{e}$ \\ R. Pellegrini ${ }^{d, f}$ and R. Pani ${ }^{f, g}$ \\ ${ }^{a}$ Morphogenesis and tissue Engineering-SAIMLAL Department, Sapienza University, Rome, Italy \\ ${ }^{b}$ Department of Physics, Sapienza University, Rome, Italy \\ ${ }^{c}$ Department of Physics and INFN, University of Roma Tre, Rome, Italy \\ ${ }^{d}$ Department of Molecular Medicine, Sapienza University, Rome, Italy \\ ${ }^{e}$ Department of Radiological Oncological and Anatomopathological Sciences, Sapienza University, \\ Rome, Italy \\ ${ }^{f}$ INFN Roma I Section, Rome, Italy \\ ${ }^{g}$ Department of Sciences and Medical-Surgical Biotechnologies, Sapienza University, Rome, Italy \\ E-mail: claudia.polito@uniroma1.it
}

ABSTRACT: The growing interest for new scintillation crystals with outstanding imaging performances (i.e. resolution and efficiency) has suggested the study of recently discovered scintillators named CRYO18 and CRYO19. The crystals under investigation are monolithic and have shown enhanced characteristics both for gamma ray spectrometry and for Nuclear Medicine imaging applications such as the dual isotope imaging. Moreover, the non-hygroscopic nature and the absence of afterglow make these scintillators even more attractive for the potential improvement in a wide range of applications.

These scintillation crystals show a high energy resolution in the energy range involved in Nuclear Medicine, allowing the discrimination between very close energy values. Moreover, in order to prove their suitability of being powerful imaging systems, the imaging performances like the position linearity and the intrinsic spatial resolution have been evaluated obtaining satisfactory results thanks to the implementation of an optimized algorithm for the images reconstruction.

Keywords: Gamma camera, SPECT, PET PET/CT, coronary CT angiography (CTA); Gamma detectors (scintillators, CZT, HPG, HgI etc)

\footnotetext{
${ }^{1}$ Corresponding author.
} 


\section{Contents}

1 Introduction 1

2 Materials and methods $\quad 2$

2.1 Materials 2

2.2 Methods 2

3 Results and discussions 3

4 Conclusion $\quad 8$

\section{Introduction}

In the last years, there was a great proliferation of new scintillation materials. Among the great variety of scintillation crystals, those with enhanced characteristics to obtain high quality images in Nuclear Medicine, are quite limited. The ideal scintillators would have a combination of several properties (like high energy and spatial resolution, refraction index near 1.5, non-hygroscopicity. .. ). Unfortunately, all the commercially available scintillators show just some of these features. As example, NaI:Tl and CsI:Tl [1] have great radiation detection properties but slow decay time and hygroscopic nature, while GYGAG [2] is not hygroscopic but with a low decay time. On the other hand $\mathrm{LaBr}_{3}$ : $\mathrm{Ce}[3]$ shows a fast decay time and excellent energy resolution against its high hygroscopicity.

Facing to this scenario, the aim of this work is to present some interesting imaging features of new scintillation crystals, named CRYO18 and CRYO19, developed by Crytur company [5]. These scintillator materials are declared without afterglow and hygroscopic nature (it means that they do not require a sealed casing that could influence spatial resolution and position linearity), with low refraction index, medium density and high light output $[4,5]$. Indeed, looking to the new applications in Nuclear Imaging, the fast decay time makes these crystals ideal candidates for small size detectors, allowing the coupling with silicon photomultipliers.

Moreover, another interesting application concerns the multi-isotope imaging diagnosis. This technique is based on the simultaneous administration of radioisotopes in order to study different metabolic processes acquiring more information about the organ metabolism. Usually, the clinical practice involves two radioisotopes characterized by photon emissions very close in energy (i.e. $81 \mathrm{keV}$ and $122 \mathrm{keV}$ ) [6, 7]. As a consequence, a high energy resolution is needed in order to reach a good discrimination of the information coming from the different radioisotope contributions in the overall spectrum.

In this article we report on the imaging properties of the CRYO18 and CRYO19 scintillation crystals with round shape and white painted surfaces (instead of the absorbent treated crystals dedicated for imaging). Both scintillators have been tested with a Multi Anode PhotoMultiplier Tube (MAPMT) equipped with a SBA photocathode (guaranteeing a higher quantum efficiency). In 
this way, the position linearity as a function of the energy and the dual isotope imaging performances have been evaluated.

\section{Materials and methods}

\subsection{Materials}

CRYO18 and CRY019, monolithic scintillation crystals manufactured by Crytur company are obtained by mixing rare earth silicate crystals and, according to the company declaration, they are non hygroscopic (this means that they do not require a sealed casing that could influence the spatial resolution and the position linearity response), without afterglow, with low refraction index (1.79 for CRYO18 and 1.81 for CRYO19), same light output, short decay time and same emission wavelength [5]. The crystals have white painting on the overall surfaces, round shapes and they are identical both in size and in thickness (52 $\mathrm{mm}$ diameter and $6.25 \mathrm{~mm}$ thickness) in order to couple them to a multi anode MAPMT Hamamatsu H10966A-100. Usually, scintillation crystals edges for imaging applications are black painted in order to enhance the position linearity reducing the light reflection while the white painting (also on the edges) is a typical feature for spectrometric applications (increasing the light collection) [7, 8]. On the other hand, light absorption typically causes a decrease in pulse height uniformity response and a local energy resolution degradation close to crystal edges. So, in order to reach good energy resolution performances, planar crystals with white painted edges have been chosen.

The multi anode MAPMT Hamamatsu H10966A-100 has 64 independent anodes (anodic size $6 \times 6 \mathrm{~mm}^{2}$, active area $49 \times 49 \mathrm{~mm}^{2}, 38.7 \%$ quantum efficiency at $380 \mathrm{~nm}$, typical gain $0.27 \times 10^{6}$ ), a SuperBiAlkali (SBA) photocathode, in order to guarantee a higher quantum efficiency, and it presents a reduced number of dynode stages to avoid saturation effects due to the intense scintillation light pulse [9].

The 64 output signals from the MAPMT are read out by a 64 independent channels readout electronics in order to collect the charge coming from each anode of the MAPMT for every scintillation event. All the anodes are independently read and digitalized (with 14 bit ADCs) with a maximum sampling frequency of $250 \mathrm{ksample} / \mathrm{second}$. The event selection is made by the digital control implemented in the FPGA, that analyzes the data read in each time slot and discards those below a certain threshold [10].

\subsection{Methods}

Some measurements have been performed aimed at highlight the principal points of investigation: the evaluation in terms of intrinsic parameters (in particular energy and spatial resolution), the dual-isotope spectrometry and imaging, the position linearity.

Energy resolution is a very important parameter for a detector allowing to reach outstanding performances in energy discrimination. The energy resolution is achieved by scanning the crystals surface in correspondence of each anode center utilizing a double $2.5 \mathrm{~mm}$ collimated sources $\left({ }^{133} \mathrm{Ba}-{ }^{57} \mathrm{Co}\right.$ for $C R Y 018$ and ${ }^{57} \mathrm{Co}-{ }^{137} \mathrm{Cs}$ for CRY019). To determine the energy resolution, the Full Width at Half Maximum (FWHM) of each peak has been evaluated. In order to improve the energy resolution by the reduction of the diffuse contribution in the pulse height, the Region of 
Interest (ROI) method has been employed. After a spot irradiation on the scintillation crystals, the pulse height shows the contribution of the full energy peak but also a diffuse contribution, due to the presence of the lead collimator or other scattering source, degradating the energy resolution. The ROI method allows to overcome this effect: starting from the image related to the spot irradiation under analysis, it is possible to consider just a selection on the image corresponding to the source in order to obtain a new pulse height spectrum in which only the scintillation events that contribute to the selected area on the image are considered. In the new spectrum, the contribution of the full energy peak is enhanced respect to the one of diffusion, as a consequence energy resolution performances are improved.

Another important feature for a detector, in order to ensure the great quality of images, is the spatial resolution. The limit of spatial resolution achievable by the detector, without the contribution of the collimator, is the intrinsic spatial resolution (iSR). The iSR depends to the Point Spread Function (PSF) that is the FWHM of the scintillation light spread striking the photocathode. The experimental evaluation of the iSR has been obtained by a spot irradiations on the crystal surfaces. The used radioactive source has to be thin collimated, in order to reproduce the effect of a point source. The crystal surfaces have been scanned with a $1 \mathrm{~mm}$ collimated source of ${ }^{241} \mathrm{Am}(60 \mathrm{keV}$ photon energy) for CRYO18 and a $1 \mathrm{~mm}$ collimated source of ${ }^{99 \mathrm{~m}} \mathrm{Tc}$ (140 keV photon energy) for CRYO19.

Moreover, imaging on planar crystals is strictly dependent to the position linearity, particularly if there are white painting surfaces (in favor of the spectrometry). Each crystal has a position linearity related to its intrinsic light distribution. The ideal linearity is defined as the full correspondence between the real position of a scintillation event and the reconstructed one. Unfortunately, the white painted surfaces give rise to a compression at the crystal edges, due to the presence of light distribution tails which are reflected inwards. As a consequence, a wrong position estimation could arise reducing the position linearity range. Taking into account the problems encountered, a new reconstruction algorithm Raise To Power (RTP) is utilized [11]. This algorithm is based on the standard centroid method [12] but differs from it because the charge collected by each anode is raised to power and a threshold is applied, after an electronic offset subtraction. In this way, the algorithm works on the light distribution shape reducing the charge spread and improving the iSR and the position linearity [11]. The position linearity and the dual isotope imaging performances have been evaluated scanning the crystals surface with double $2.5 \mathrm{~m}$ collimated sources $\left({ }^{133} \mathrm{Ba}-{ }^{57} \mathrm{Co}\right.$ for $C R Y 018$ and ${ }^{57} \mathrm{Co}-{ }^{137} \mathrm{Cs}$ for CRYO19) in correspondence of each anode center.

\section{Results and discussions}

In figure 1, the pulse height of $C R Y 018$ and CRO19, obtained with the ROI method, are shown. The spectra are related to a spot irradiation at the center of the crystals with a double $2.5 \mathrm{~mm}$ collimated source $\left({ }^{133} \mathrm{Ba}-{ }^{57} \mathrm{Co}\right.$ for $C R Y 018$ and ${ }^{57} \mathrm{Co}-{ }^{137} \mathrm{Cs}$ for CRYO19). The full energy peaks of the various emissions are well recognizable at low $(32 \mathrm{keV}, 81 \mathrm{keV}, 122 \mathrm{keV})$ and high $(662 \mathrm{keV})$ energies. Moreover, the full energy peak contributions are enhanced respect to the ones of diffusion and, as a consequence, the energy resolution performances are improved. Energy resolution is $15.7 \%$ for CRYO18 and $18.2 \%$ for CRYO19@122 keV. These results are related to the spot irradiation at the crystal center.

In figure 2, the importance of the new position algorithm for the crystals is well demonstrated. The image is related to a scan on the CRYO19 surface with a $2.5 \mathrm{~mm}{ }^{57} \mathrm{Co}$ collimated source (the 

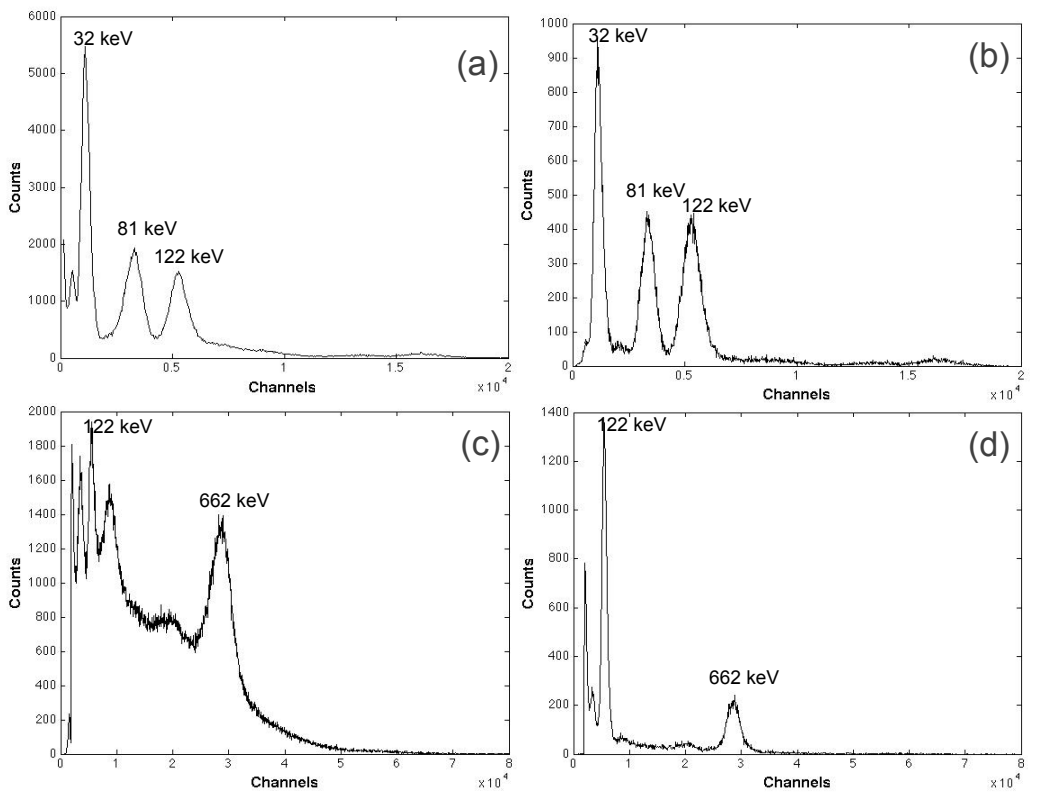

Figure 1. Pulse height spectra of double $2.5 \mathrm{~mm}$ collimated source ${ }^{133} \mathrm{Ba}-{ }^{57} \mathrm{Co}$ for $C R Y 018$ without (a) and with (b) ROI selection. Pulse height spectra of double $2.5 \mathrm{~mm}$ collimated source ${ }^{57} \mathrm{Co}-{ }^{137} \mathrm{Cs}$ for CRYO19 without (c) and with (d) ROI selection. All the pulse height spectra are related to the spot irradiation at the crystal center.

experimental scan distance is $6 \mathrm{~mm}$ ). With the standard Anger method (that correspond to the power $=1$ ) the Field of View (FoV) is limited, because of the light tails that are reflected inwards causing a degradation in the reconstruction of the scintillation event positions. By increasing the power in RTP algorithm, the FoV size is almost recovered reaching a satisfactory result comparable with the ones obtained with similar detectors [7]. Images in figure 2 show a halo due to the presence in CRY019 of a self activity background of ${ }^{176} \mathrm{Lu}$ [4]. The halo is accentuated on the crystal edges because of the light reflections due to the white painted surfaces.

The position linearity is obtained from the scanning of the $C R Y O 18$ surface with a $2.5 \mathrm{~mm}{ }^{57} \mathrm{Co}$ collimated source and the scanning of the CRYO19 surface with a $2.5 \mathrm{~mm}{ }^{137} \mathrm{Cs}$ collimated source (the experimental scan distance is always $6 \mathrm{~mm}$ ).

The evaluation of the influence of RTP method on the linearity allows to demonstrate that a crystal with an expected flat linearity (due to the white painted surfaces), is able to become a powerful imaging system thanks to the application of a new reconstruction algorithm that overcomes Anger's logic and allows to recover position linearity.

Figure $3 \mathrm{a}$ and figure $3 \mathrm{~b}$ highlight this behavior for CRY018 and CRY019 respectively: by increasing power in the reconstruction algorithm, position linearity becomes close to the theoretical one. So, increasing the power, the FoV compressions are reduced and the position linearity becomes better (same results have been obtained for CRYO18).

However, a compromise between the FoV increment and the power has to be reached. In fact, a too high power causes a consistent cut off of the bottom contribute of the light distributions reaching a FoV that is close to the real one but degrading the image uniformity (making the anodic pattern slowly visible). For this reason the adequate power in RTP algorithm is 2 or 2.5. Moreover, the 

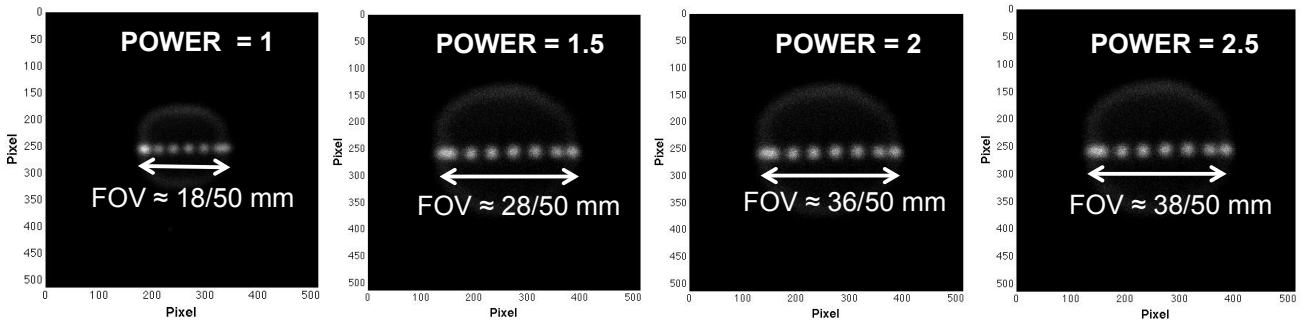

Figure 2. Images coming from the scanning of CRYO19 surface with $2.5 \mathrm{~mm}{ }^{57}$ Co collimated source $(6 \mathrm{~mm}$ step) as a function of the utilized power.

comparison of the position linearity of both crystals in (figure 3c) shows that they have the same spatial linearity expecially at the center of the crystals (where there are not compression effects due to the reflections on the edges). This suggests that despite the different mixture of both scintillators, they have the same optical properties in terms of light transport.

Table 1. iSR results for CRYO18 and CRYO19 scintillation crystals as a function of the power in the reconstruction algorithm. The values are obtained from spot irradiations with a $1 \mathrm{~mm}$ collimated source of ${ }^{241} \mathrm{Am}$ for $C R Y 018$ and a $1 \mathrm{~mm}$ collimated source of ${ }^{99 \mathrm{~m}} \mathrm{Tc}$ for CRYO19.

\begin{tabular}{|c|c|c|}
\hline & Power $=1$ & Power $=2$ \\
\hline CRY018 & $1.8 \mathrm{~mm}$ & $1.7 \mathrm{~mm}$ \\
CRY019 & $2.5 \mathrm{~mm}$ & $1.9 \mathrm{~mm}$ \\
\hline
\end{tabular}
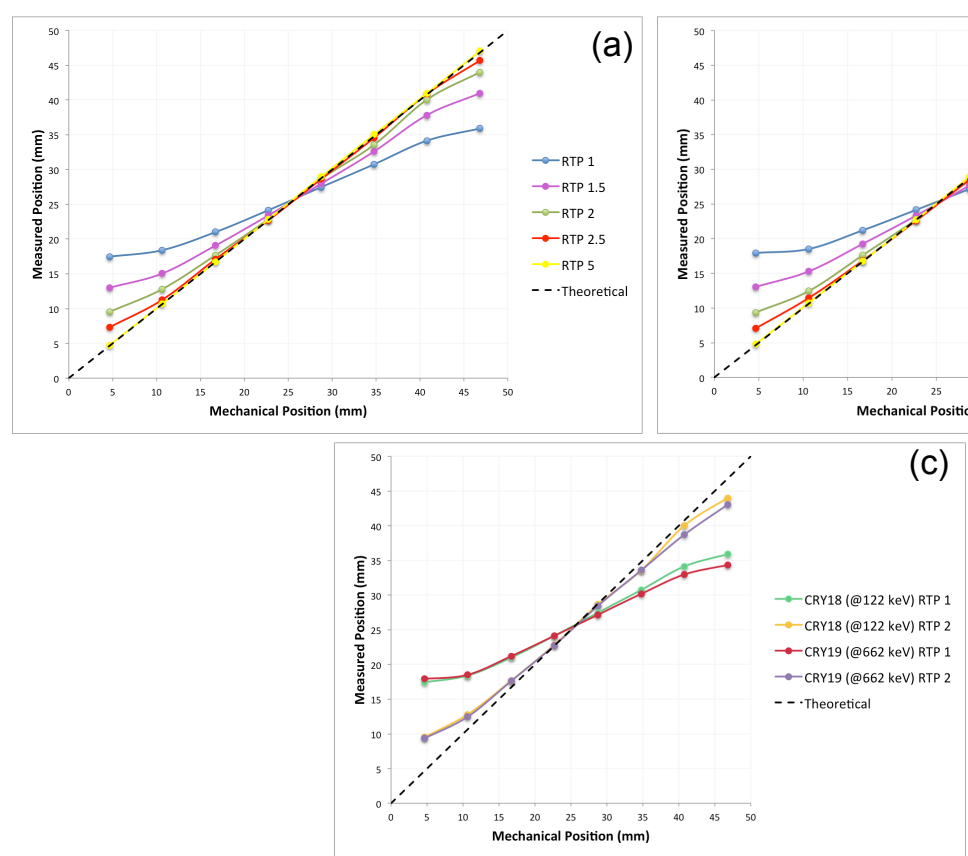

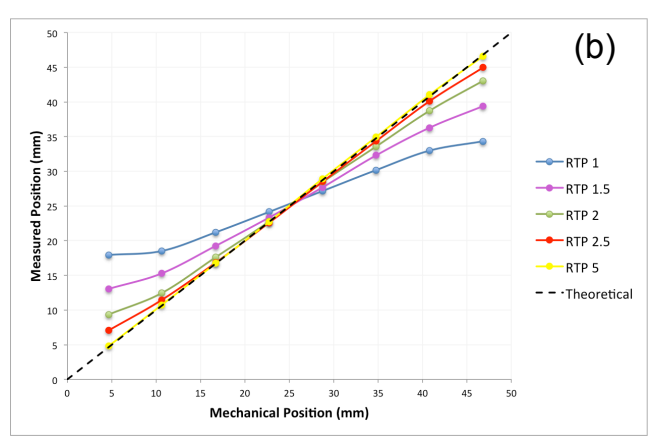

(c)

(n)

Figure 3. Position linearity evaluated from spot irradiations for $C R Y O 18$ (a) and $C R Y 019$ (b) using different powers. Comparison of the $C R Y 018$ and $C R Y 019$ position linearity (c) for power $=1$ and power $=2$. 
The position linearity allows to define a pixel $/ \mathrm{mm}$ conversion correlating the position of the spot in the image pixel with the mechanical position of the spot in mm. Finally, the iSR has been evaluated from the FWHM of the spot profile (subtracting the contribution of the source collimator). Regarding the experimental evaluation, the iSR for CRYO18 has been evaluated scanning the crystal surface with a $1 \mathrm{~mm}$ collimated source of ${ }^{241} \mathrm{Am}$ in correspondence of each anode center, as shown in figure 4 (while the CRYO19 surface has been scanned with a $1 \mathrm{~mm}$ collimated source of ${ }^{99 \mathrm{~m}} \mathrm{Tc}$ ).

The spot irradiation in figure 4 shows a visible compression at the edges. This behavior is due to the experimental setup, indeed the round crystal has been assembly on a square MAPMT and the dimensions mismatch (as discussed in the previous section the crystal diameter $(52 \mathrm{~mm})$ is greater than the MAPMT side $(49 \mathrm{~mm})$ ). As consequence, the image suffers from this assembly and this is particularly evident at the angles (when the crystal is not superimposed on the MAPMT). Moreover, the white painted surfaces have a relevant influence on the imaging producing internal light reflections at the edges and causing a further reduction of the position linearity.

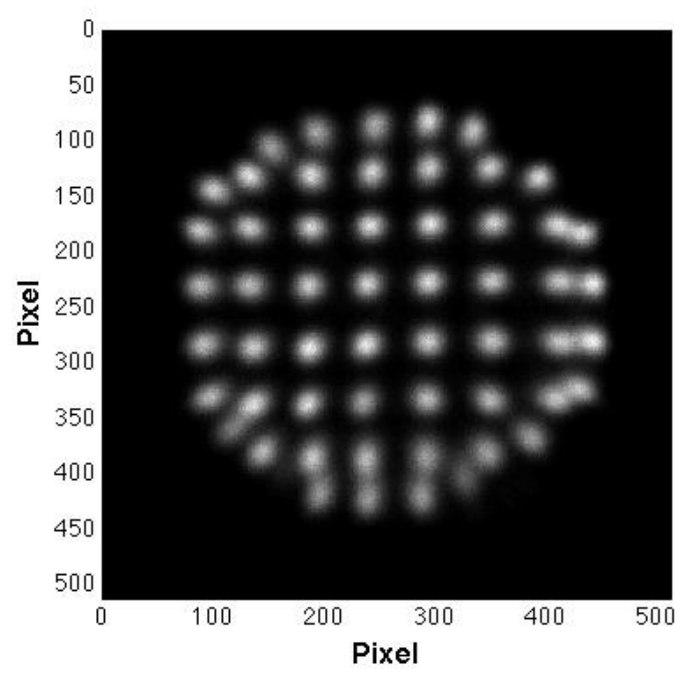

Figure 4. Image of $C R Y 018$ spot irradiation on each anode center with $1 \mathrm{~mm}$ collimated source of ${ }^{241} \mathrm{Am}$. Image reconstructed with RTP algorithm (power $=2$ ).

In table 1 the values of iSR for both crystals are shown. The reported iSR values are the mean values obtained from the spots along the crystals diameter. As expected, increasing the power in the image reconstruction algorithm, the iSR improves respect to the values obtained with the standard centroid method (corresponding to power $=1$ ).

In order to further demonstrate the outstanding imaging performances of CRYO18 and CRYO19 scintillation crystals, the results of dual isotope measurements on both crystals are shown. In figure 5 dual isotope results for $C R Y 018$ are presented in terms of pulse height distributions, total image and images in ${ }^{133} \mathrm{Ba}$ and ${ }^{57} \mathrm{Co}$ energy windows, respectively @ $81 \mathrm{keV}$ and @ $122 \mathrm{keV}$, for 1:1 uptake and for the sources positioned at the center of the crystal. Images are reconstructed with RTP algorithm (power $=2$ ). The employed sources are sealed with the same external diameter but with the internal one slightly different. In this way, even if the sources are piled upon each other in order to be used simultaneously, they have a small geometrical distance of about $0.2 \mathrm{~mm}$ that results correctly detected by the detection system and that has been estimated from the centroid evaluation. 
Unfortunately, for the spot irradiation at the crystal edge this geometrical distance is not equally well detected due to the position linearity losses in that area.
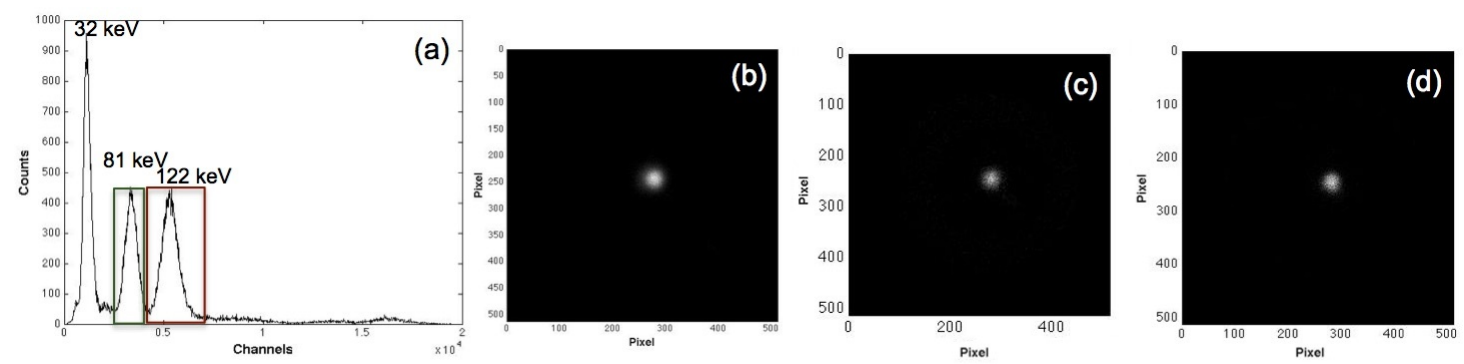

Figure 5. Dual isotope imaging for CRY018. (a) Dual isotope pulse height distribution. (b) Dual isotope overall image. (c) Image in ${ }^{133} \mathrm{Ba}$ energy window (green area in the pulse height distribution). (d) Image in ${ }^{57}$ Co energy window (red area in the pulse height distribution).

In figure 6 dual isotope results for $C R Y 019$ are presented in terms of pulse height distributions, total image and images in ${ }^{57} \mathrm{Co}$ and ${ }^{137} \mathrm{Cs}$ energy windows, respectively @ $122 \mathrm{keV}$ and @ $662 \mathrm{keV}$, for 1:1 uptake and for the sources positioned at the center of the crystal. Images are reconstructed with RTP algorithm (power $=2$ ). In the images in figure 6 the halo due to the self activity background is still recognizable. In particular, the shadow is well evident in figure $6 \mathrm{~d}$ due to the relevant amount of self activity counts in the ${ }^{137} \mathrm{Cs}$ energy range while in the ${ }^{57} \mathrm{Co}$ energy window (figure 6c) the halo is always present but not quite visible due to the lower amount of self activity counts in that energy window for the evaluation of just one spot.
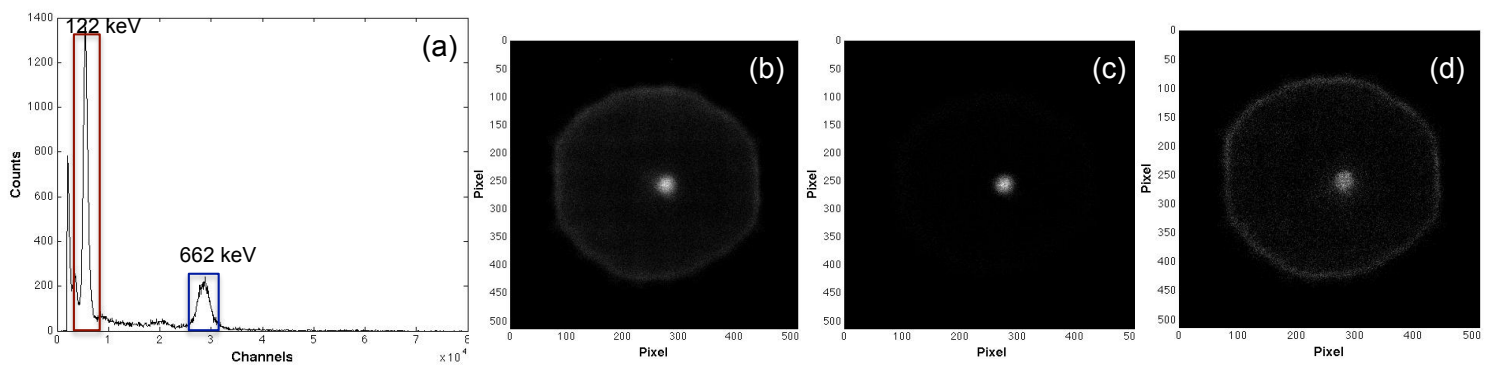

Figure 6. Dual isotope imaging for CRY019. (a) Dual isotope pulse height distribution. (b) Dual isotope overall image. (c) Image in ${ }^{57} \mathrm{Co}$ energy window (red area in the pulse height distribution). (d) Image in ${ }^{137} \mathrm{Cs}$ energy window (blue area in the pulse height distribution).

Images of the radioactive sources are obtained by selecting the appropriate energy window in the pulse height spectrum. The possibility to discriminate the different contribution with high precision demonstrate the efficiency of the employed methods and the satisfactory performances in terms of energy resolution values. In fact CRY018 shows satisfactory results compared with the energy resolutions values of the in use scintillation crystals in the SPECT energy range $[1,4,13]$ and the CRY019 too, with an energy resolution of about 7.6\%@662 keV shows the best energy resolution value compared to the others lutetium based scintillators [4, 14]. In this way, is possible to well discriminate the imaging information coming from two sources separately. This result, in 
addition with the ones obtained by the imaging characterization previously described, demonstrates how improvements in spatial and energy resolution allow to improve Nuclear Medicine imaging.

\section{Conclusion}

Nuclear Medicine imaging provides useful procedures aimed at the diagnosis of different diseases. In this contest, the high quality of images is an essential aspect. Therefore, an upgrade of the detectors performances is needed. This improvement is strictly correlated to the spatial and energy resolutions of the imaging devices.

In this work, improvement in imaging detectors based on recently developed scintillation crystals have been presented. In spite of the white painting at all sides, the spatial resolution performances of CRYO18 and CRYO19 scintillation crystals are satisfactory. This results have been achieved with the introduction of a new position reconstruction algorithm that overcomes the intrinsic limitation of the Anger logic and works on the reduction of the light tail contributions, that are reflected inwards degrading the accuracy of the event position reconstruction. In this way, crystals with high energy resolution performances due to the spectrometric features can be used for imaging applications, without degradation of the spatial resolution.

The enhanced performances of these scintillators allow to reach excellent results also in dual isotope applications. The imaging from radioisotopes with different photon emissions (i.e. ${ }^{111} \mathrm{In}$, ${ }^{201} \mathrm{Tl},{ }^{75} \mathrm{Se}$ ) is a useful and often performed technique for cerebral and cardiac imaging but also for many others applications as the preoperative localization of parathyroid tumors [6]. So, an accurate discrimination of the different contribution in the overall spectrum can increase considerably the diagnostic potential.

In the next future, taking advantage from features like the non hygroscopic nature and the short decay time, these crystals could be also employed in other applications as the dual modality imaging.

\section{References}

[1] P. Lecoq, Development of new scintillators for medical applications, Nucl. Instrum. Meth. A 809 (2016) 130.

[2] A. Giaz, Characterization of new scintillators $\mathrm{SrI}_{2}: \mathrm{Eu}, \mathrm{CeBr} r_{3}, \mathrm{GYGAG}: \mathrm{Ce}$ and CLYC:Ce, J. Phys. Conf. Ser. 620 (2015) 012003.

[3] M.N. Cinti, R. Pani, R. Pellegrini, P. Bennati, C. Orlandi, A. Fabbri et al., Spectrometric performances of high quantum efficiency multi and single anode PMTs coupled to $\mathrm{LaBr}_{3}(\mathrm{Ce})$ crystal, Nucl. Instrum. Meth. A 724 (2013) 27.

[4] R. Pani, M. Colarieti-Tosti, M.N. Cinti, C. Polito, C. Trigila and S. Ridolfi, Investigation of radiation detection properties of CRY-018 and CRY-019 scintillators for medical imaging, 2016 JINST 11 P09010.

[5] http://www.crytur.cz/materials/.

[6] D.R. Neumann, Simultaneous Dual-Isotope SPECT Imaging for the Detection and Characterization of Parathyroid Pathology, J. Nucl. Med. 33 (1992) 131. 
[7] R. Pani et al., LaBr 3 :Ce small FOV gamma camera with excellent energy resolution for multi-isotope imaging, 2015 JINST 10 C06002.

[8] S. Lo Meo et al., Geant4 simulation for modelling the optics of $\mathrm{LaBr}_{3}: \mathrm{CE}$ scintillation imagers, IEEE Nucl. Sci. Symp. Conf. Rec. (2008) 4964.

[9] http://www.hamamatsu.com/eu/en/product/alpha/P/3002/H10966A/index.html.

[10] A. Fabbri et al., Dual isotope imaging with LaBr 3 :Ce crystal and H8500 PSPMT, 2013 JINST 8 C02022.

[11] R. Pani et al., New position arithmetic for scintillation camera based on floating weight system, IEEE Nucl. Sci. Symp. Conf. Rec. (2011) 3395.

[12] C. Martin et al., Wedge and strip anodes for centroid finding position-sensitive photon and particle detectors, Rev. Sci. Instrum. 52 (1981) 1067.

[13] T. Zeniya et al., Use of compact pixellated gamma camera for small animal pinhole SPECT imaging, Ann. Nucl. Med. 20 (2006) 409.

[14] R. Pani et al., A study of a response of a response of a LuYAP:Ce array with innovative assembling for PET, Nucl. Instrum. Meth.A 795 (2015) 82. 